\title{
An Algorithm for the Global Solution of the Shape-from-Shading Model
}

\author{
M. Falcone and M. Sagona \\ Dipartimento di Matematica, Università di Roma "La Sapienza", \\ P. Aldo Moro 2, 00185 Roma, e-mail: falconeecaspur.it
}

\begin{abstract}
A numerical scheme to solve the Dirichlet type problem for the first order Hamilton-Jacobi equation related to the shape-fromshading model is proposed. The algorithm computes the maximal soIution of the problem provided a compatibility condition on the discretization steps is satisfied. This global formulation allows to include in the model the informations brought by the shadows in a rather natural way avoiding cumbersome boundary conditions on the interfaces between light and shadows and the use of additional informations on the surface.
\end{abstract}

\section{Introduction}

Let $S$ be a Lambertian surface given as a graph $z=u(x), x \in \mathbb{R}^{2}$ and let us assume that there is a unique light source at infinity whose direction is indicated by the unit vector $\omega=\left(\omega_{1}, \omega_{2}, \omega_{3}\right) \in \mathbb{R}^{3}$. We will assume in the sequel that $\omega$ is known. As it is well known, (see f.e. [6]) the partial differential equation related to the Shape-from-Shading (SFS) model can be derived by the Image Radiation Equation

$$
R(\hat{n}(x))=I(x)
$$

where $I(x)$ is the brightness function measured at all points $x$ in the domain of $u, \hat{n}(x)$ is the unit normal to the surface at the point $(x, u(x))$ and $R(\hat{n}(x))$ is the reflection map giving the value of the light reflection on the surface as a function of its orientation (i.e. of the normal) at each point. The brightness function $I$ is known in the model since it is measured on each pixel of the "picture" for example in terms of a grey level (from 0 to 255). To construct a continuous model we will assume that $I$ takes (real) values in the interval $[0,1]$.

Assuming that $u$ has a compact support $\Omega$ and recalling that for a Lambertian surface $R(\hat{n}(x))=\hat{n}(x) \cdot \hat{\omega}$ equation (1) can be written in the form

$$
I(x) \sqrt{1+|\nabla u(x)|^{2}}+\left(\omega_{1}, \omega_{2}\right) \cdot \nabla u(x)-\omega_{3}=0, \text { for } x \in \Omega
$$

which is a first order nonlinear partial differential equation of Hamilton-Jacobi type. Moreover, we complement the equation with the natural Dirichlet boundary condition

$$
u(x)=0 \quad \text { for } x \in \partial \Omega
$$

* This work was supported by the EEC Program Human Capital "Mathematical Modelling of Image Processing" (Contract ERB CHRX-CT93-0095) 
which corresponds to the assumption that the surface is standing on a background. The solution of the above Dirichlet problem (2), (3) will give the surface corresponding to the brightness $I(x)$ measured in the "picture" representing $\Omega$.

There are several technical difficulties related to this problem. The first is that the surface can be non differentiable at some points so that we have to consider solutions in a "weak sense". The theory of viscosity solutions for Hamilton-Jacobi type equations provides the right framework for the analysis of the problem (see [4] for an up-to-date presentation of that theory) although to get uniqueness, the problem is usually solved adding some informations such as the height at the points where the brightness has a maximum or the complete knowledge of a level curve (see e.g. [10]). Recent results of the theory of viscosity solutions allow to characterize the maximal solution without extra informations besides the equation and to construct an algorithm which converges to that solution. From the numerical point of view one would like to have an algorithm able to compute non smooth solutions.

The addition of "shadows" makes the problem even more complicated since in principle the equation is defined only where $I(x)>0$ and the interfaces between light and shadows could be non regular curves. The approach briefly described here allows to compute a global solution with just one single computation and does not require any preliminary reconstruction of the light/shadow interfaces (for a more detailed analysis of the algorithm we refer to [7]).

It should be noted that other global algorithms have been proposed in [9]. They use different numerical schemes to solve the associated Hamilton-Jacobi equation (f.e. the finite difference scheme introduced in [11]) and they do not include shadows as we are.

\section{Approximation of the maximal solution of the eikonal equation}

Let us start examining the theoretical results available for the case of a vertical light source. That case corresponds to $\left(\omega_{1}, \omega_{2}, \omega_{3}\right)=(0,0,1)$ so the general equation (2) becomes

$$
I(x) \sqrt{1+|\nabla u(x)|^{2}}-1=0, \quad x \in \Omega
$$

Writing (4) in explicit and adding the homogeneous Dirichlet boundary condition we obtain

$$
\begin{cases}|\nabla u(x)|=f(x), & x \in \Omega \\ u(x)=0 & x \in \partial \Omega\end{cases}
$$

where

$$
f(x)=\sqrt{\frac{1}{I(x)^{2}}-1} \quad 0<I(x) \leq 1 .
$$

Note that with a vertical light we will never have shadows since our surface is a graph. This implies that $I(x)$ can only vanish at some single points. Moreover, 
$f(x)=0$ where $I(x)=1$, i.e. the right-hand side vanishes at all points of maximum brightness and this causes the lack of uniqueness of "classical" viscosity solutions. However, a result by Ishii-Ramaswamy [8] gives a characterization of the maximal solution of (2), (3) for the eikonal equation corresponding to the case of a vertical light source.

In order to construct a numerical approximation, let us observe first that the eikonal equation (4) can also be written as

$$
\begin{cases}\max _{a \in B_{2}(0,1)}\{-a \cdot \nabla u(x)\}=f(x), & x \in \Omega \\ u(x)=0 & x \in \partial \Omega\end{cases}
$$

since the maximum will be achieved for $a^{*} \equiv-\nabla u(x) /|\nabla u(x)|$.

Here and in the sequel we will assume for simplicity that $u \geq 0$. This is not restrictive, since the equation just depends on $\nabla u$ and we can always add to $u$ the constant $u_{0} \equiv \min _{x \in \Omega}\{u(x)\}$ to satisfy that requirement. In order to obtain an approximation scheme in the form of a fixed point problem it is useful to introduce the new variable

$$
v(x)=1-e^{-u(x)}
$$

Note that by definition $0 \leq v \leq 1$. The problem for the new variable $v$ is

$$
\begin{cases}v(x)+\max _{a \in B_{2}(0,1)}\left\{-\frac{a}{f(x)} \cdot \nabla v(x)-1\right\}=0, & x \in \Omega \\ v(x)=0 & x \in \partial \Omega\end{cases}
$$

where $f$ is given by (6).

It is known (see e.g. [1]) that (9) has a unique continuous viscosity solution provided $f$ is bounded and never vanishes in $\Omega$.

Let us introduce the fully discrete scheme. To simplify, we will assume that the "picture" is a rectangle $\Omega \subset R^{2}$. Let us consider a mesh of the set $\Omega_{\delta}=$ $\Omega+\delta B_{2}(0,1)$. We will denote by $\mathcal{I}_{i n}$ the set of indices of the nodes $x_{i}$ belonging to $\Omega$, by $\mathcal{I}_{\text {out }}$ the set of indices of the nodes belonging to $\Omega_{\delta} \backslash \Omega$ (where we will impose the boundary condition) and by $\mathcal{I}$ their union. We will also denote by $N_{\text {in }}, N_{\text {out }}$ and $N$ respectively the number of nodes belonging to $\Omega_{\text {in }}, \Omega_{\text {out }}$ and to their union.

Let $k$ be the size of the mesh and let $W^{k}$ denote the space of piecewise affine functions which are linear on the cells ( $P^{1}$ finite element approximation). We look for a solution $w \in W^{k}$ of

$$
\begin{gathered}
w\left(x_{i}\right)=\min _{a \in B_{2}(0,1)}\left\{\mathrm{e}^{-h} w\left(x_{i}+h \frac{a}{f\left(x_{i}\right)}\right)\right\}+\left(1-\mathrm{e}^{-h}\right), \text { for } i \in \mathcal{I}_{i n} \\
w\left(x_{i}\right)=0, \text { for } i \in \mathcal{I}_{\text {out }} .
\end{gathered}
$$


In [5] it has been proved that the numerical solution of (10) exists and it is unique. The numerical solution of our problem can be computed by a fixed point iteration on the operator $T: \mathbb{R}^{N} \rightarrow \mathbb{R}^{N}$

$$
(T(V))_{i} \equiv \begin{cases}\min _{a \in B_{2}(0,1)}\left\{\mathrm{e}^{-h} \Lambda(a) V\right\}_{i}+1-\mathrm{e}^{-h}, & i \in \mathcal{I}_{\text {in }} \\ 0 & i \in \mathcal{I}_{\text {out }}\end{cases}
$$

where $V$ is the $\mathrm{N}$-dimensional vector containing the values at the nodes of the mesh, i.e. $V_{i}=v\left(x_{i}\right)$, and $\Lambda(a)$ is the matrix of the local coordinates of the points $x_{i}+h \frac{a}{f\left(x_{i}\right)}$.

It is easy to prove that the operator $T$ is a contraction mapping and a monotone operator from $[0,1]^{N}$ to $[0,1]^{N}$ so that there exists a unique fixed point $V^{\star}, V^{\star}=T\left(V^{\star}\right)$. By the monotonicity property starting from a subsolution $\left(V_{0} \leq T\left(V_{0}\right)\right)$ the sequence will monotically converge to the fixed point. This property is crucial to speed-up convergence and it also helps to compute the maximal solution.

\section{Approximation of the maximal solution with shadows}

Let us go back to our general equation corresponding to a light source in the direction of $\omega$. If the light is oblique we will have shadows so that we can divide the support of the surface (the domain of $u$ ) into two regions,

$$
\Omega_{l} \equiv\{x: I(x)>0\}, \quad \Omega_{s} \equiv\{x: I(x)=0\}
$$

which represent respectively the "light" and the "shadow" regions. Naturally, $\Omega=\Omega_{l} \cup \Omega s$ and we will assume that also the projection of the shadows on the background will fall in $\Omega$.

In $\Omega_{l}$ the equation is always the same, whereas in the "shadow" region the surface can have any shape since the model is not able to describe the real surface there. This is why other authors have included boundary conditions (f.e. Neumann boundary conditions) on $\partial \Omega_{l}$ to treat the problem in $\Omega_{l}$ just ignoring the region $\Omega_{s}$. This can in turn create some difficulties in the construction of the numerical algorithm since the boundary of $\Omega_{l}$ can be non smooth and it will not belong to the mesh (unless a special mesh is constructed starting from that boundary).

Following [3] we include the region $\Omega_{s}$ in the computation just defining there a conventional surface to replace the (unknown) surface. We will substitute to the surface the "separation ray" (or "shadow ray") i.e. the ray separating light from shadows. That ray has the same direction of $\omega$. This means that in $\Omega_{s}$ we have to solve the equation

$$
\left(\omega_{1}, \omega_{2}\right) \cdot \nabla u(x)-\omega_{3}=0, \quad x \in \Omega_{s}
$$

Note that (2) coincides with (14) since $I=0$ in $\Omega_{s}$. Then, we can use the same equation everywhere in $\Omega$ and we will not need to introduce any boundary condition on $\Omega_{l}$, i.e. we can write the global problem as 


$$
\left\{\begin{array}{lr}
I(x) \sqrt{1+|\nabla u(x)|^{2}}+\left(\omega_{1}, \omega_{2}\right) \cdot \nabla u(x)-\omega_{3}=0, & x \in \Omega \\
u(x)=0 & x \in \partial \Omega
\end{array}\right.
$$

Following the same lines of Section 2, we obtain the fully discrete scheme

$w\left(x_{i}\right)=\min _{a \in B_{3}(0,1)}\left\{\beta w\left(x_{i}+h b\left(x_{i}, a\right)\right)-(1-\beta) \frac{I\left(x_{i}\right) a_{3}}{\omega_{3}}\left(1-w\left(x_{i}\right)\right)\right\}+1-\beta$

where $\beta=e^{-h}$ and $b(x, a)=\frac{1}{\omega_{3}}\left(I(x) a_{1}-\omega_{1}, I(x) a_{2}-\omega_{2}\right)$.

The new operator $T_{s}$ corresponding to the oblique light source is

$$
\left(T_{s} u\right)(x) \equiv \min _{a \in B_{3}(0,1)}\left\{\beta u(x+h b(x, a))-(1-\beta) \frac{I(x) a_{3}}{\omega_{3}}(1-u)\right\}+1-\beta
$$

Under appropriate conditions, the operator $T_{s}$ has the same properties of $T$ and the algorithm will converge monotonically to the maximal solution.

On the boundary we just impose the homogeneous Dirichlet boundary condition. This condition implies that the shadows must not cross the boundary of $\Omega$, so the choice $\omega_{3}=0$ corresponding to an infinite shadow behind our surface is not admissible. For the analysis of the algorithm and for some hints on its implementation we refer to [7].

\section{$4 \quad$ Numerical experiments}

The experiments has been made using curves and surfaces with different types of regularity (just continuous or differentiable) and with different numbers of maximum brightness points. In the vertical and in the oblique case we have made a cut-off on $I$ at the level $\bar{I}$, defining the new brightness function

$$
\widehat{I}(x)= \begin{cases}I(x) & \text { if } 0 \leq I(x)<\bar{I} \\ \bar{I} & \text { if } \bar{I} \leq I(x) \leq 1\end{cases}
$$

The cut-off is necessary in the vertical light case since when $I(x)=1$ the vectorifield "blows-up" at the points of maximum brightness. As far as the choice of the discretization steps is concerned, we observe that the convergence to the maximal solution takes place when $k$ and $h$ are such that $\frac{k}{h} \geq 1$.

Let us consider some 2-dimensional surface reconstructions with vertical light source. In the first example we have reconstructed a piramid. In that case the brightness image (Fig. 1b) has a unique grey level. The reconstruction is represented in Figure 1a, it has been obtained with $\Delta x=\Delta y=h=0.05, \bar{I}=0.99$ and gives an $L^{1}$ error of about 0.0195 . As a second example we considered a smooth surface. Figures $1 \mathrm{c}, 1 \mathrm{~d}$ and $1 \mathrm{e}$ respectively show the real solution surface, the brightness image and the maximal solution. In this case the image has five internal maximum points where we have to make a cut-off (here $\bar{I}=0.96$ ). The numerical solution has been computed for $\Delta x=\Delta y=0.02$ and $h=0.01$. Figure if is the numerical reconstruction of the same surface obtained fixing 
the height at the maximum brightness points as in [12]. We conclude with the reconstruction of a real grey-level image representing a vase (Figure 2a). Figure $2 \mathrm{~b}$ shows the computed surface for $\Delta x=\Delta y=h=0.01$ and $\bar{I}=0.99$. It is interesting to compare the original image with the images obtained computing $I$ from the computed surface. Infact, once we have the surface we can compute $I\left(x_{i j}\right)$ at each node $x_{i j}$ simply making the scalar product $\omega \cdot \eta\left(x_{i j}\right)$. However, note that the numerical solution has been computed over a grid so that the normal is not uniquely defined at each node. In principle, there are four admissible normals corresponding to the triangles having $x_{i j}$ among their vertices and several (non equivalent) choices are possible. Figure $2 \mathrm{c}$ shows the intensity obtained taking the normal of the upper rightmost triangle. In that case one can note that the numerical surface is non smooth along three curves. One can also compare the original image with the one in Figure 2c obtaining an $L^{1}$ error is about 0.0082 . Taking all the possible normals (four as we said) one can compute four different values of the intensity at every internal node $x_{i j}$. Figure $2 \mathrm{~d}$ shows the image corresponding to the minimum of those values at each node, the $L^{1}$ error is about 0.0076 . In that case the picture looks smoother and closer to the original.

\section{References}

1. M. Bardi and M. Falcone, An approximation scheme for the minimum time function, SIAM Journal of Control and Optimization, 28 1990, 950-965.

2. F. Camilli, Qualche applicazione delle soluzioni viscosità a problemi di evoluzione di fronti, Ph. D. Thesis, Dipartimento di Matematica dell'Università di Roma "La Sapienza", October 1995.

3. F. Camilli and M. Falcone, An approximation scheme for the maximal solution of the shape-from-shading model, Proceedings ICIP 96 International Conference on Image Processing, IEEE Inc., 1996, 49-52.

4. M. G. Crandall, H. Ishii and P. L. Lions, User's guide to viscosity solutions of second order partial differential equations, Bull. Amer. Math. Soc., 27 (1992), 1-67.

5. M. Falcone, The minimum time problem and its applications to front propagation, in A. Visintin e G. Buttazzo (eds), "Motion by mean curvature and related topics", De Gruyter Verlag, Berlino, 1994

6. B.K.P. Horn and M.J. Brooks, Shape from Shading, The MIT Press, 1989.

7. M. Falcone and M.Sagona, forthcoming.

8. H. Ishii and M. Ramaswamy, Uniqueness results for a class of Hamilton-Jacobi equations with singular coefficients, preprint, 1995.

9. R. Kimmel and A.M. Bruckstein, Global Shape fron Shading, Center for Intelligent System Report CIS \#9327,Technion-Israel Inst. of Tech., Israel, November 1993.

10. P.L. Lions, E. Rouy and A. Tourin, Shape from shading, viscosity solution and edges, Numerische Mathematik, 64, (1993), 323-353.

11. S.J. Osher and J.A. Sethian, Fronts Propagating with curvature dependent speed: Algorithms based on Hamilton-Jacobiformulations, J. of Comput. Phys., 79 (1988), $12-49$.

12. E. Rouy and A. Tourin, A viscosity solutions approach to Shape from Shading, SIAM J. Numer. Anal., 29 (1992), 867-884. 

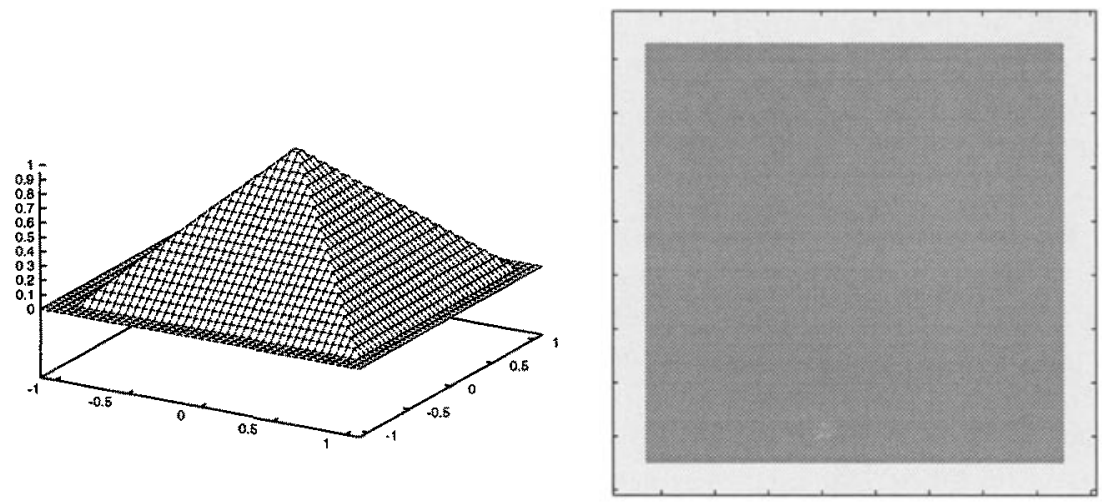

$1 a$

lb

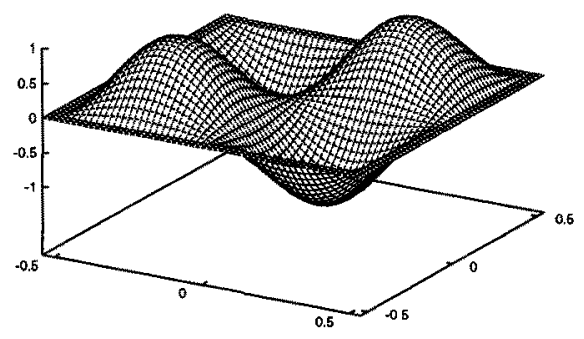

$1 \mathrm{c}$

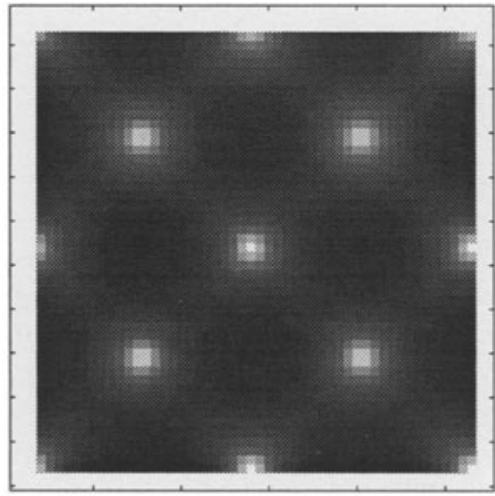

$1 d$
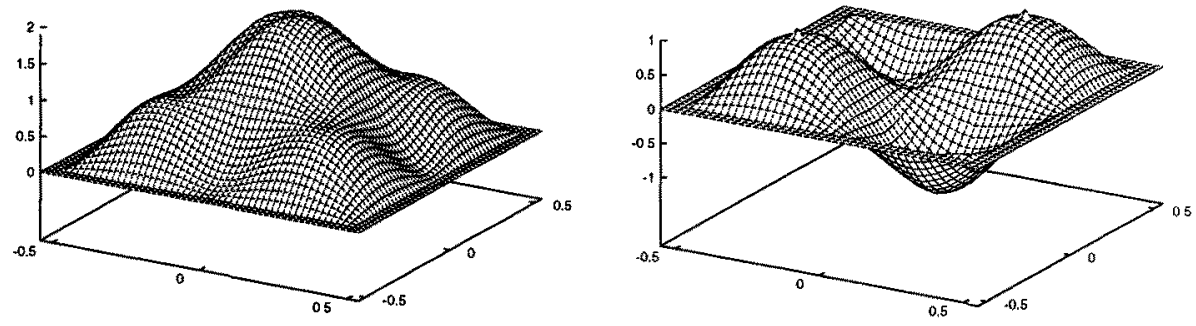

$1 \mathrm{e}$ 


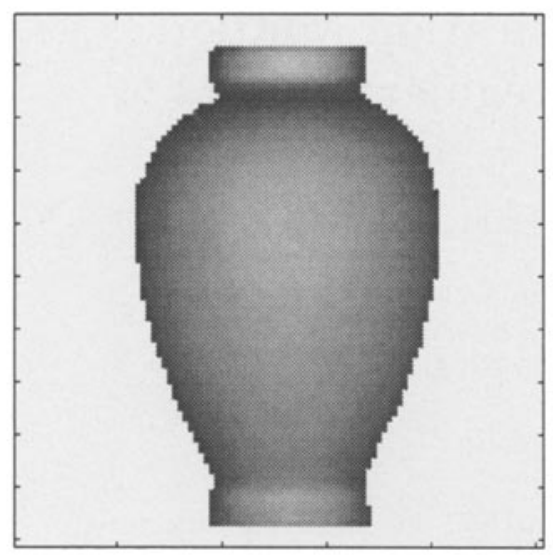

$2 a$

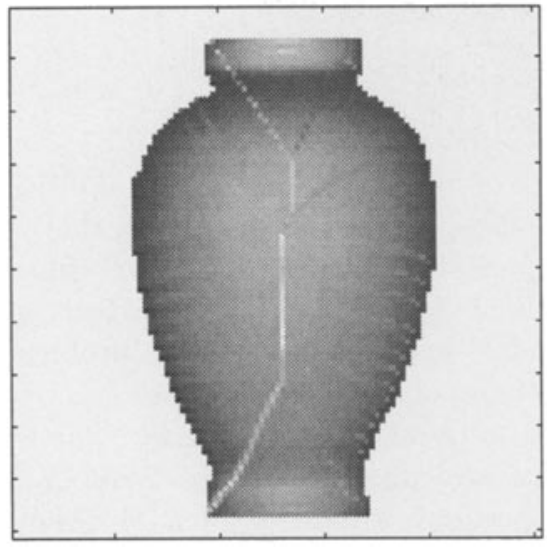

$2 c$

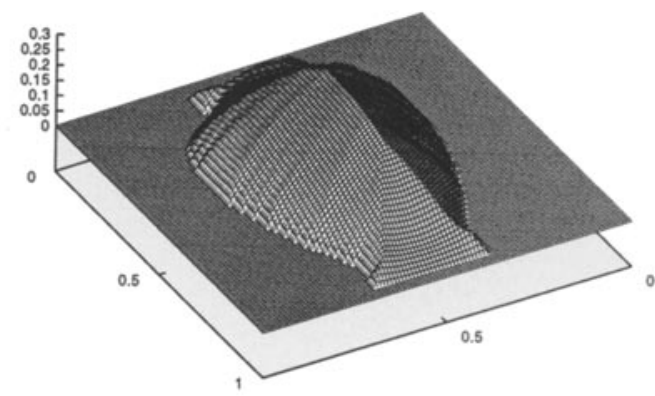

$2 b$

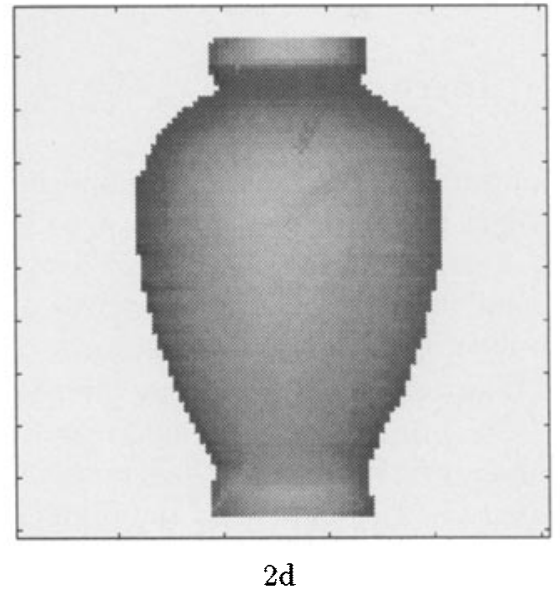

